\title{
Las pioneras en exigir educación científica: Ciencia, mujer y prensa en el Chile decimonónico ${ }^{1}$
}

\section{The pioneers in demanding scientific education: Science, woman and press in Chile (19th Century)}

\author{
Verónica Ramírez *
}

\begin{abstract}
Resumen
Este artículo revisa desde una perspectiva histórica los esfuerzos protagonizados por una red de mujeres chilenas durante las últimas tres décadas del siglo XIX, destinados a conseguir su derecho a educarse científica y profesionalmente. Su enfoque se centra en el análisis de publicaciones efectuadas por estas mujeres en la prensa, lo que a su vez responde a una actitud rupturista que les permitió tomarse espacios negados antes para ellas (la ciencia y la prensa), yendo más allá de lo establecido, y motivando con sus acciones reformas y cambios sociales profundos. La tesis principal desarrollada es que la irrupción de la mujer en la prensa chilena está completamente ligada a esta red de intelectuales que exigió el derecho de educarse profesional y científicamente. Se postula que esta última convicción fue el aliciente para que ellas se tomaran aquel espacio público, convirtiéndose la prensa en la herramienta principal para lograr sus objetivos.
\end{abstract}

Palabras clave: Educación, ciencia, mujeres, prensa, Chile, siglo XIX.

\footnotetext{
${ }^{1}$ Este capítulo fue desarrollado en el marco del proyecto FONDECYT Postdoctoral № 3180131 , titulado "Astronomía y literatura en Chile: diálogo y discusión en el espacio público (1880-1930), actualmente en ejecución.

* Doctora en Literatura chilena e hispanoamericana, académica e investigadora de la Facultad de Artes Liberales, Universidad Adolfo Ibáñez. Sus investigaciones se han enfocado en el diálogo entre el discurso literario y científico presente en la prensa chilena del siglo XIX y principios del XX, centrándose entre otras perspectivas, en la situación y rol de las mujeres en dicho contexto. Entre sus publicaciones relacionadas con el presente trabajo se destaca el libro Antología crítica de mujeres en la prensa chilena del siglo XIX (2017) y La Mujer (1877): primer periódico de mujeres en Chile (2018).
} 


\begin{abstract}
This article reviews from a historical perspective the efforts carried out by a network of Chilean women during the last three decades of the nineteenth century, aimed at achieving their right to educate themselves scientifically and professionally. Its focus is on the analysis of publications made by these women in the press, which in turn responds to a rupturist attitude that allowed them to take previously denied spaces for them (science and the press), going beyond the established, and motivating with its actions reforms and deep social changes. The main thesis that develops is that the irruption of women in the Chilean press is completely linked to this network of intellectuals who demanded the right to educate themselves professionally and scientifically. It is postulated that this last conviction was the incentive for them to take that public space, making the press the main tool to achieve their objectives.
\end{abstract}

Keywords: Education, science, women, press, Chile, 19th Century.

Fecha de recepción: Enero 2019

Fecha de aprobación: Diciembre 2019

\title{
1. Introducción.
}

La discusión sobre la educación científica y profesional de las mujeres en nuestro país, como dice Ana María Stuven (2010), se inicia junto con la fundación de la Universidad de Chile en 1842. A partir de ese momento, se instala la pregunta de si era conveniente para la sociedad que las mujeres siguiesen estudios superiores y, por ende, si era necesario que ellas recibieran formación científica. Este debate, que puede constatarse en las páginas de la prensa nacional, fue liderado hasta la década de 1860 por políticos, intelectuales, docentes y religiosos varones, pero desde 1870 en adelante las mujeres tomarán la palabra y protagonizarán la defensa de una educación científica para ellas ${ }^{2}$.

En 1871, Eduvigis Casanova, directora de la Escuela Superior de Valparaíso, publicó su texto Educación de la mujer, inaugurando así una nueva etapa de este debate, que a partir de entonces será protagonizado por las propias mujeres. Un año después apareció la traducción realizada por Martina Barros Borgoño del estudio crítico de John Stuart Mill, titulado La esclavitud de la mujer, donde aboga por la educación femenina.

2 En 1844, en un comunicado publicado en el semanario El Alfa de Talca, una mujer que firma como Una del sexo -seudónimo atribuido a Carmen Arriagada- reflexiona sobre las deficiencias de la educación femenina en su ciudad, pero expresa claramente que no justifica una educación científica para la mujer (El Alfa, No.7, Talca, 14 de diciembre de 1844). En el periódico La Mariposa, que circuló en Valparaíso entre 1863 y 1864, aparece un discurso de una alumna del colegio de las señoritas Cabrera que se pronuncia sobre la educación de la mujer, pero no se refiere a la preocupación por las materias científicas (La Mariposa, No. 17, Valparaíso, 20 de enero de 1864). Pueden encontrarse otras publicaciones como estas antes de la década de 1870, pero en ellas no se encuentra una defensa hacia la educación científica de las mujeres. Recién en la década de 1870 ellas se manifiestan públicamente a favor de esto último. 
Ese mismo año Antonia Tarragó, directora del Colegio Santa Teresa, hizo la primera solicitud formal a la Comisión Universitaria para que sus alumnas pudieran validar los exámenes secundarios y postular a la universidad ${ }^{3}$. En 1873 Rosario Orrego se convirtió en la primera directora de un periódico en Chile, y entre los objetivos principales de este se encontraba la difusión científica. Dos años después se fundó en San Felipe La Brisa de Chile, primer proyecto periodístico dedicado especialmente a la ilustración de las mujeres, y en el cual se intensificó el debate liderado por las intelectuales en pro de la instrucción y profesionalización de la mujer ${ }^{4}$. En 1876, Isabel Le Brun, directora del Colegio de La Recoleta, volvió a solicitar -ahora con mayor éxito- lo mismo que cuatro años antes Tarragó no había podido conseguir. Así, en febrero de 1877, el Ministro de Instrucción Pública, Miguel Luis Amunátegui, firmó el decreto que permitió a las chilenas acceder a los estudios universitarios. En directa relación con este acontecimiento, ese año cobró vida el periódico La Mujer, cuya redactora principal fue la intelectual liberal Lucrecia Undurraga. Las páginas de este último se convirtieron en un relevante espacio para reflexionar sobre la Ley Amunátegui, así como para mantener la atención del público en otras problemáticas que debían resolverse para que la normativa pudiese ser aplicada satisfactoriamente ${ }^{5}$. Como puede evidenciarse, la década de 1870 fue un periodo crucial para la discusión y avance en materia de derechos sociales de las mujeres chilenas, proceso que fue cubierto y orquestado por la prensa.

El decreto del Ministro de Instrucción Pública afirmó legalmente que las mujeres sí podían desarrollarse profesionalmente y, en consecuencia, que estaban facultadas para instruirse científicamente. Esta Ley, no obstante, consistió en un primer gesto para la mejora de la educación femenina, ya que tras esta medida faltarían muchísimos otros factores a modificar para que realmente fuera factible el acceso de las mujeres al campo profesional y científico en nuestro país. Lo relevante es que el debate desarrollado en la prensa antes, durante y después del decreto de 1877, impulsó la conformación y sostenimiento en el tiempo, de una red de mujeres que se ocuparía durante las siguientes décadas del siglo XIX de defender y asegurar la concreción favorable de dicha iniciativa.

La irrupción de la mujer en la prensa chilena, por consiguiente, está completamente ligada a esta red de intelectuales que exigió el derecho de educarse profesional y científicamente. Esta última convicción fue el aliciente para que ellas utilizaran la prensa como herramienta principal para lograr sus objetivos. Se debe tener en cuenta que las mujeres en 1870 no tenían derecho a voto, no podían ejercer cargos políticos, ni representación legal, por lo que para concientizar a la ciudadanía de que ellas sí debían educarse y sí podían seguir una carrera profesional, acudieron estratégicamente a la prensa. Por lo tanto, nuestro trabajo pretende revisar desde una perspectiva histórica los esfuerzos protagonizados por esta red de mujeres. Nuestro análisis se enfoca en las publicaciones efectuadas por ellas en periódicos que circularon durante las últimas décadas del XIX, cuyo carácter rupturista les permitió entrar en espacios negados antes

\footnotetext{
${ }^{3}$ Esta solicitud no tuvo respuesta satisfactoria por parte de las autoridades.

${ }^{4}$ Para profundizar en este periódico, véase: Ramírez, Romo y Ulloa, 2017.

${ }^{5} \mathrm{Si}$ se quiere profundizar sobre este periódico, véase: Ramírez, Romo y Ulloa, 2017.
} 
para ellas, en este caso, el mundo científico y profesional, yendo más allá de lo establecido, y motivando con sus acciones reformas y cambios sociales profundos.

La perspectiva histórica se enfrenta en este trabajo desde varias miradas teóricas. Una de ellas es la historia de la ciencia, sobre todo de autores que se han dedicado al análisis de la comunicación de la ciencia y que consideran la transmisión del conocimiento científico tan relevante como la producción de dicho conocimiento. James Secord (2004) postula que la ciencia es un acto comunicativo per se, por lo que es imposible separar la producción de ciencia de la comunicación de esta. En este sentido, las mujeres que se pronunciaron sobre asuntos científicos en el XIX, habrían contribuido en el desarrollo de esta área del saber. Esta mirada también se vincula a las ideas de otros autores, tales como Bruno Latour (1987) y Lipphardt y Ludwig (2011), que entienden a la ciencia como un proceso y no como un producto acabado, en el que además interactúan varios grupos y espacios de la sociedad, y no solo los llamados "expertos", como reafirman Agustí Nieto-Galan (2011) y Jürgen Renn (2015), entre otros.

El enfoque histórico de este trabajo también se enmarca desde una perspectiva de género, y para ello debe tenerse en cuenta que la relación entre ciencia y género ha sido controversial. Como han planteado Bowler y Rhys Morus (2005), la crítica feminista de los años 60 y 70 desarmó la fachada que la ciencia se construyó a sí misma como modelo de objetividad, revelando la importancia que han tenido en su desarrollo la clase, las ideologías religiosas y el género, entre otros. Esta mirada permite comprender algunas lógicas que contribuyeron a la conformación de la ciencia, fundamentándose en un análisis del pasado desde una mirada crítica que considera la influencia de las identidades de género en el desarrollo de la ciencia. La historiografía ha mostrado el vínculo entre ciencia y género en los últimos siglos, exponiendo cómo ello ha marcado a sus actores, espacios y lenguajes, entre otros. Como ha señalado Donald Opitz (2015), por ejemplo, la ciencia moderna se nutrió del espacio doméstico y de los roles desempeñados en él para la producción del conocimiento científico. De esta manera, se ha incorporado a nuevos agentes de producción científica, poniendo en entredicho la idea de que la ciencia solo se hace desde los laboratorios mediante los llamados expertos. Por lo que, a partir de estas nuevas miradas, se ha comenzado a tomar en cuenta a sujetos periféricos de los espacios que tradicionalmente se instalaron como los lugares de generación del saber, permitiendo una conceptualización de la ciencia más heterodoxa y diversa.

Como el análisis recae en fuentes escritas por mujeres en la prensa, también es necesario considerar un enfoque histórico que aplique una perspectiva de género a la práctica de la escritura y al uso del lenguaje como un modo de desenvolverse en el espacio público. Para ello es apropiado considerar el trabajo seminal de Josefina Ludmer (1985), debido a que las pioneras en exigir educación científica a través de la prensa chilena utilizaron, entre otras estrategias discursivas, las "tretas del débil". Asimismo, resulta apropiado dialogar con la relación entre escritura y género que desarrollan las primeras escritoras feministas en nuestro país, tal como lo ha estudiado Andrea Kottow (2013). Finalmente, creemos pertinente leer nuestras fuentes a la luz de lo que propone Nancy Fraser (1992) respecto al espacio público presentado por Habermas. La autora, 
criticando la democracia actualmente existente, repiensa la esfera pública y, además, desarrolla el concepto de "contra-públicos", señalando que los subordinados o excluidos construyen espacios propios.

La metodología de trabajo ha consistido, primero, en establecer ciertos parámetros para seleccionar las fuentes. El estudio previo de Claudia Montero (2018) sobre la historia de la prensa escrita por mujeres en el Chile decimonónico, así como el trabajo anterior de nuestra autoría (Ramírez, Romo y Ulloa, 2017), nos permitió revisar 66 periódicos, quedándonos con un corpus específico para el análisis actual, centrado en los proyectos de los últimos 30 años del siglo XIX en que las mujeres tuvieron un rol protagónico, tanto como colaboradoras, así como editoras y directoras, y en aquellos donde la educación científica y profesional de las mujeres fue tema relevante. Los principales periódicos considerados en nuestro análisis, según dicho criterio, son los que aparecen en la tabla 1.

Tabla 1. Listado de proyectos periodísticos mayormente contemplados para el análisis.

\begin{tabular}{|c|c|c|c|c|c|}
\hline Periódico & Inicio & Término & Lugar & Producción & Lectores \\
\hline $\begin{array}{l}\text { Revista de } \\
\text { Valparaíso }\end{array}$ & 1873 & 1874 & Valparaíso & $\begin{array}{l}\text { Directora mujer; } \\
\text { colaboradores } \\
\text { hombres y mujeres }\end{array}$ & $\begin{array}{l}\text { Público } \\
\text { heterogéneo }\end{array}$ \\
\hline $\begin{array}{l}\text { La Brisa de } \\
\text { Chile }\end{array}$ & 1875 & 1876 & San Felipe & $\begin{array}{l}\text { Director/a } \\
\text { anónimo/a; } \\
\text { colaboradores } \\
\text { hombres y mujeres }\end{array}$ & $\begin{array}{l}\text { Público } \\
\text { preferentemente } \\
\text { femenino }\end{array}$ \\
\hline La Mujer & 1877 & 1877 & Santiago & $\begin{array}{l}\text { Directora mujer; } \\
\text { colaboradoras } \\
\text { mujeres en su } \\
\text { mayoría }\end{array}$ & $\begin{array}{l}\text { Público } \\
\text { preferentemente } \\
\text { femenino }\end{array}$ \\
\hline La Familia & 1890 & 1892 & Santiago & $\begin{array}{l}\text { Directora mujer; } \\
\text { colaboradoras } \\
\text { mujeres en su } \\
\text { mayoría }\end{array}$ & $\begin{array}{l}\text { Público } \\
\text { preferentemente } \\
\text { femenino }\end{array}$ \\
\hline
\end{tabular}

La lista no incluye todos los proyectos dirigidos y principalmente producidos por mujeres dentro del periodo, ya que, insistimos, nos hemos detenido en aquellos donde la exigencia de la educación científica y profesional de ellas fue una temática central. Así mismo, con el objetivo de no excluir otros textos relevantes sobre la causa firmados por mujeres, hemos revisado la prensa diaria -cuya producción es de dominio de varones-, así como otros periódicos en que se incluyeron excepcionalmente colaboraciones femeninas, como es el caso de Revista de Santiago (1872-1873) y Biblioteca Republicana (1894), entre otros.

Es relevante señalar que la búsqueda de algunas de estas fuentes fue extremadamente compleja, sobre todo para el caso del periódico La Mujer de 1877, dirigido por Lucrecia Undurraga, ya que no se encontraba ningún ejemplar ni en archivos públicos, ni privados, por lo que gran parte de nuestra metodología tuvo que volcarse hacia el hallazgo de este documento y hacia su resguardo patrimonial. 


\section{La prensa y el Decreto Amunátegui.}

Los alegatos postulados por mujeres para exigir el derecho a educarse de manera similar a los hombres, como hemos adelantado, fueron orquestados por las reflexiones que las intelectuales publicaron en la prensa nacional a lo largo de toda la década de 1870, logrando posicionar este debate como uno de los temas principales en el espacio público. La solicitud al Consejo Universitario realizada por Antonia Tarragó en 1872 se fundamentó en el decreto promulgado ese año que autorizaba la toma de estos exámenes a los alumnos de colegios particulares. Antes, para ser válidos, estos solo podían rendirse en el Instituto Nacional. Si los estudiantes de colegios particulares masculinos contarían con ese derecho a partir de entonces, ¿por qué no podían gozar de este también las alumnas de colegios femeninos? Esta era la pregunta que se hacía la directora del Colegio Santa Teresa en Santiago. Su solicitud se imprimió y circuló entre los círculos académicos, políticos y de la alta sociedad, pero no hubo respuesta positiva de parte del Estado.

Esta indiferencia se contrapuso, sin embargo, al eco producido por su solicitud en la prensa y al efecto motivador que condujo a otras mujeres a pronunciarse también. Ese mismo año, Martina Barros Borgoño (sobrina de Diego Barros Arana) se atrevió a traducir el famoso estudio del filósofo inglés, John Stuart Mill, titulado The Subjection of Women, y publicarlo, junto a un prólogo de su autoría, en Revista de Santiago. En sus páginas la autora se tomó la licencia de comentar y discutir sobre distintos puntos del trabajo del renombrado inglés, así como fortalecer el argumento iniciado por Antonia Tarragó. La censura que recibió por este acometido, no obstante, se dejó caer con fuerza, a pesar de las precauciones que ella tomó en el mismo texto, cuando señalaba, por ejemplo, que "El título de este libro pudiera hacerlo aparecer con un alcance sedicioso que no tiene" (Barros, 1872: 112).

Pese a la censura, la participación en la prensa protagonizada por mujeres, así como el sostenimiento del debate sobre su educación científica, aumentó en esos años. En 1873 y 1874, Rosario Orrego dirigió Revista de Valparaíso, en la cual además de publicarse textos sobre el problema de la educación de la mujer, algunas escritoras se pronunciaron sobre materias científicas, convirtiéndose en las primeras difusoras de la ciencia - propiamente tal- en nuestro país (Ramírez, 2016). Esta revista señalaba entre sus objetivos principales la transmisión del saber, por lo que el papel ejercido por esta intelectual- tanto de directora de un periódico, como de editora de textos de difusión científica- fue doblemente subversivo en la época.

Siguiendo el mismo impulso, y como ya hemos adelantado en el apartado anterior, en 1875 cobró vida un nuevo proyecto periodístico, que se impuso como propósito central ilustrar a las lectoras y reflexionar sobre el problema de la educación de la mujer. Nos 
referimos a La Brisa de Chile, semanario sobre literatura, ciencias, artes, modas, música y variedades, que circuló en San Felipe. Entre el material publicado en sus páginas, destaca un nuevo texto de Eduvigis Casanova, titulado "Reflexiones sobre la educación pública de la mujer en Chile: Escuelas públicas de niñas". En este expuso el estado actual de ese segmento educativo y propuso reformas, tales como la creación de comisiones de señoras para fomentar el amor a la instrucción, la moralidad y el trabajo (Casanova, 1876: $\mathrm{s} / \mathrm{p})$.

Como ya mencionamos, en 1876 Isabel Le Brun, directora de un colegio privado femenino, presentó una nueva solicitud ante el Consejo Universitario. El requerimiento fue publicado íntegramente en distintos periódicos nacionales, tales como La República, El Independiente, El Ferrocarril, El Deber y El Mercurio. Los efectos que tuvo esta petición fueron cubiertos por estos medios durante los últimos meses de 1876 y los primeros días de 1877, desatándose un debate de gran envergadura que obligó al Ministro de Instrucción Pública a firmar el famoso Decreto Amunátegui el 6 de febrero de 1877. La Ley versaba así:

"Considerando: 1) que conviene estimular a las mujeres a que hagan estudios serios y sólidos; 2) que ellas pueden ejercer con ventaja, algunas de las profesiones denominadas científicas; y 3) que importa facilitar los medios de que puedan ganar la subsistencia por sí mismas, Decreto: se declara que las mujeres deben ser admitidas a rendir exámenes válidos para obtener títulos profesionales, con tal que se sometan para ello a las mismas pruebas a que están sujetos los hombres"(Amunátegui, 1877: 34).

Después de la autorización otorgada por el gobierno, el clamor de la opinión pública amainó, pero en ningún caso cesó, ya que surgieron nuevas inquietudes. ¿Qué otros elementos debían desarrollarse para que esta nueva ley pudiera llevarse a cabo? ¿Existían las facilidades para que las mujeres efectivamente pudiesen acudir a la universidad? Estas y otras preocupaciones fueron sostenidas en la prensa desde 1877, poniendo en entredicho que el Decreto de Amunátegui fuese efectivamente viable. La falta de liceos públicos de niñas demostraba que aún no había de parte del Estado un compromiso concreto hacia la educación de las chilenas. En este contexto se fundó un nuevo periódico de suma importancia para el debate en cuestión. Nos referimos al ya mencionado La Mujer de 1877, dirigido por Lucrecia Undurraga y escrito casi exclusivamente por mujeres (Ulloa, 2017: 122-130) ${ }^{6}$. Este semanario comenzó a circular en mayo de 1877 y en su prospecto se explica claramente cuál será la idea que sustentará todos sus números: "Empeñar todos nuestros esfuerzos en mejorar la situación actual de la mujer; abogar sin tregua ni descanso por sus intereses bien entendidos; levantar sus aspiraciones hasta las luminosas esferas del saber" (Undurraga, 1877: 1). En las páginas de los veinticinco números subyace la convicción de que el mejoramiento social de las mujeres depende de su educación y profesionalización.

${ }^{6}$ En sus veinticinco números solo se encuentran siete colaboraciones firmadas por varones. 
Un dato relevante que nos ofrece este último periódico es la existencia real de una red de mujeres intelectuales a lo largo del país, vinculada a su vez con personalidades y organizaciones extranjeras. Las colaboradoras de La Mujer (1877) vivían en diversas localidades de Chile, destacándose Santiago, Valparaíso, Copiapó y San Felipe como ciudades en las cuales existía una mayor concentración de escritoras comprometidas con la causa. Entre estas también había extranjeras, tales como las españolas Gertrudis Gómez de Avellaneda y Concepción Gimeno de Flaquer, y la argentina Juana Manuela Gorriti, todas promotoras de reformas y mejoras sociales para las mujeres (Ramírez y Ulloa, 2018).

El acompañamiento que brindó la prensa a este cometido continuó también durante las siguientes décadas. La preocupación por la educación profesional de las mujeres, así como por el lugar que debían ocupar estas en el campo científico, se mantuvo en publicaciones periodísticas posteriores a 1877, complementado, a partir de entonces, con una mayor cantidad de textos sobre diversos asuntos científicos firmados por mujeres.

En enero de 1885, casi dos años antes de que se titulara la primera mujer de la Universidad de Chile ${ }^{7}$, Maipina de la Barra, ejerciendo como corresponsal del periódico chileno La Patria, envió desde Europa un texto que describía el terremoto acontecido en Granada (España) el 25 de diciembre de 1884. En su colaboración no solo señaló efectos sociales ocasionados por el movimiento telúrico, sino que también reflexionó sobre las causas mismas de este fenómeno, entregando registros exactos sobre las particularidades de los siguientes sismos (De la Barra, 1885). De la Barra, en consecuencia, se manifestó públicamente sobre un asunto científico en términos técnicos, siguiendo el camino iniciado por las difusoras de Revista de Valparaíso en 1873. En 1889 María Luisa Acevedo Lay, ex alumna del Colegio de Isabel Le Brun y dedicada posteriormente a la docencia y dirección de establecimientos educacionales públicos, envió al periódico chileno El Progreso su texto "Sobre los estudios que convienen a la mujer". Esta publicación era el Órgano del Club del Progreso y su objetivo era la difusión de ideas en pos del adelanto de las ciencias y el cultivo de las letras. El texto de Acevedo defendía una vez más la educación científica de la mujer (Acevedo, 1889). En septiembre de ese mismo año, La República -revista literaria dirigida por Enrique C. Latorre- publicó un discurso de Eurídice Pinochet, hija de Isabel Le Brun y alumna del colegio fundado por ella ${ }^{8}$, en el que se reflexiona sobre la vinculación que existe entre la literatura y la ciencia, pronunciándose nuevamente sobre un asunto de competencia científica (Pinochet, 1889). En 1890 se fundó la revista La Familia, cuya directora era la francesa radicada en Santiago, Celeste Lassabe. Esta publicación, dedicada especialmente al seno del hogar y, por ende, a las mujeres, señaló en el texto inicial titulado "Nuestra primera palabra", que en el periódico "tendrán cabida artículos científicos" (Lassabe, 1890: 1). En 1893 se publicó en La Verdad la colaboración de una autora que firmaba como Ulrica. Allí se expone sobre las diferencias intelectuales entre los hombres y las mujeres, defendiendo

\footnotetext{
${ }^{7}$ Nos referimos a Eloísa Díaz, quien se tituló de médico-cirujano a fines de 1886.

${ }^{8}$ Más tarde, Eurídice Pinochet sigue los pasos de su madre, dedicándose a la pedagogía.
} 
las facultades femeninas (Ulrica, 1893). En 1894, las páginas de Biblioteca Republicana incluyeron el trabajo de la peruana Margarita Práxedes Muñoz, titulado "Diferencias en el volumen craneoscópico de los dos sexos". Esta Bachiller en Ciencias por la Universidad de San Marcos y médico por la Universidad de Chile, exponía que la atribución de mayor inteligencia a los hombres era infundada desde el punto de vista biológico (Muñoz, 1894).

Estas y otras publicaciones aparecidas permanentemente en la prensa nacional desde la década de 1870, debieron influir para que en la última década del siglo XIX se concretara la fundación de los primeros liceos femeninos en el país. La defensa de la necesidad de estos establecimientos fue, de hecho, uno de los temas principales sostenidos acerca de la educación de las mujeres desde la promulgación del Decreto Amunátegui. Por lo que, de cierta manera, la escritura de estas intelectuales pudo ser responsable de que en 1891 se fundara el Instituto Carlos Waddington en Valparaíso, y en 1894, el Liceo de Niñas №1 Javiera Carrera en Santiago, forzando al Estado por fin a responsabilizarse de garantizar el ingreso de las mujeres a la universidad, es decir, de que ellas recibieran formación científica y profesional.

\section{Las protagonistas del debate.}

La batalla argumental desarrollada en la prensa a favor de una educación científica y profesional para las chilenas, fue liderada por un grupo de mujeres intelectuales que gozaron de ciertas competencias para exponer sus ideas. Eduvigis Casanova, Rosario Orrego, Antonia Tarragó, María Luisa Cerna, Isabel Le Brun, Lucrecia Undurraga, Enriqueta Courbis, Enriqueta Solar, Emilia Lisboa, María Isabel Randolph, Elena Salazar, María Luisa Acevedo y Eurídice Pinochet, son solo algunos de los nombres de quienes protagonizaron este movimiento en favor de la instrucción femenina. Entre sus ocupaciones se destacó el ejercicio docente, así como el oficio periodístico y literario, desempeñándolos de manera amateur y sin remuneración en la mayoría de los casos. Como plantean Ossandón y Santa Cruz (2005), la profesionalización del periodismo y del trabajo del escritor se produce en Chile durante los primeros años del siglo XX, gracias a la creación de empresas editoriales con propósitos comerciales, como la de Agustín Edwards Mac Clure, entre otras. Algunas pedagogas, en cambio, sí tuvieron la oportunidad de formarse en la escuela normalista fundada en 1854 y recibir pago por su desempeño, pero la mayoría de ellas recibió formación en espacios privados, ya sea en colegios particulares femeninos o mediante institutrices y de manera autodidacta en sus hogares. Solo algunas de las más jóvenes de este grupo pudieron acceder a la educación universitaria.

A pesar de que la promulgación del Decreto Amunátegui se efectuó en 1877, recién a fines de la década de 1880 se graduaron las dos primeras alumnas de la Universidad de Chile: las médico-cirujanas Eloísa Díaz y Ernestina Pérez. La falta de un sistema que apoyara la formación profesional de las mujeres, así como los prejuicios instalados en la sociedad respecto a la participación de ellas en el mundo científico y profesional (y en la vida pública en general), obstaculizaron el ingreso masivo de ellas a la 
universidad. A eso se debe sumar que la mayoría de las mujeres que siguió estudios superiores durante el siglo XIX en Chile, no optaron por seguir carreras científicas. En 1895 Chile contaba con veintinueve graduadas de Bachillerato en Filosofía y Humanidades, mientras que las profesiones netamente científicas tuvieron que esperar varios años todavía para atraer alumnas a sus aulas. Un respaldo de esto último se verifica en que recién en 1919 se graduó la primera alumna de la Escuela de Ingeniera de la Universidad de Chile (Justicia Acuña). Otro dato relevante, en este sentido, es que prácticamente todas las profesionales que lograron obtener un título o grado universitario en el país durante el siglo XIX, recibieron su enseñanza primaria y secundaria en el sistema privado, ya que, como hemos adelantado, el primer liceo público femenino se fundó recién en 1891.

El pensamiento de esta red de mujeres que protagonizó el debate en cuestión no coincidió en todos los puntos. Algunas de ellas fueron más liberales que otras, como el caso de Lucrecia Undurraga frente a las directoras de colegios particulares Antonia Tarragó e Isabel Le Brun, quienes conducían establecimientos educativos católicos. Algunas difirieron también en la idea de si las mujeres debían ejercer exactamente las mismas profesiones que los hombres. Un grupo defendió, por ejemplo, que las mujeres intelectualmente son completamente iguales a los varones, y otras, por el contrario, sostuvieron que ellas son diferentes a los hombres en ese aspecto, resaltando la importancia del carácter único de la mujer y su valor dentro de la sociedad. Este tipo de contradicciones dentro del grupo, no obstante, serán parte de la naturaleza del debate, pero no generará mayores roces o desavenencias entre las mujeres que publican en la prensa sobre la educación de la mujer. De hecho, entre ellas es posible constatar más ideas en común divergencias, manifestando sobre todo unanimidad en el derecho de las mujeres a recibir educación científica y profesional. Vinculado con esto último, es relevante destacar que la mayoría de estas voces comprendieron a la ciencia y a la filosofía como una materia que no se contraponía a la religión, posibilitando el diálogo entre intelectuales de distintas tendencias.

Otro aspecto que las unió fue el talento y vocación por la escritura que todas ellas demostraron poseer, siendo ambas cosas herramientas claves para exigir y conseguir el derecho a acceder al campo científico y profesional. A continuación, analizaremos más detenidamente la forma en que sirvieron del lenguaje para sostener este debate.

\section{Textos que marcaron hitos.}

El texto de Eduvigis Casanova, titulado "Educación de la mujer" (1871), se erige como uno de los hitos de este proceso, ya que inaugura en Chile la discusión pública sobre materia educativa protagonizada por mujeres. La publicación se inicia con la exposición de las materias que son parte del currículum de las escuelas públicas femeninas. Respecto a esto, Casanova explica que el programa es sumamente básico y que la mujer debería tener la posibilidad de recibir instrucción en otras disciplinas. La autora se pregunta: “¿Por qué no habrían de ir más adelante aquellas que recibieron del 
cielo el inestimable don de la inteligencia?” (Casanova, 1871: 20); y luego agrega: “¿Por qué no estudiarán cosmografía y astronomía, historias, idiomas extranjeros, literatura, filosofía, pintura, música, etc.?" (Casanova, 1871: 20). Para resolver estas preguntas, Casanova expone una serie de argumentos, ordenados lógicamente y valiéndose de respaldos sustentados en el sentido común. Se opone, primero, a la idea establecida de que las mujeres no deben estudiar aquellas disciplinas que no utilizarán en el futuro. En segundo lugar, rechaza el mito de que las mujeres más instruidas se han de volver más fastidiosas. $Y$, en tercer lugar, no acepta que sea negativo que ellas manifiesten públicamente su talento, tal como lo hacen los hombres.

Para enfrentar lo primero, la pedagoga explica que las mujeres deberían aprender todo tipo de materias, porque estas otorgarían herramientas a las que enviudan y deben valerse por sí mismas; añadiendo, además, que instruirlas en ramos como la astronomía sería fundamental para que estas pudiesen apoyar y potenciar los estudios de sus hijos (Casanova, 1871).

Para contraponerse al prejuicio de que la mujer instruida fastidiaba a quienes la rodeaban, plantea que:

"Nunca supo la ignorante darse el porte digno a la par que afectuoso y cordial con el cual se presenta en todas partes la que es verdaderamente instruida; nunca supo tomar el lenguaje de las diferentes edades y condiciones para agradar a todos, gozando ella misma y aprovechando del trato social de esas diferentes personas" (Casanova, 1871: 22).

Finalmente, sobre la aversión a que las mujeres muestren públicamente sus talentos, la educadora explica: "No hay ley alguna moral o civil que condene a la mujer a ocultar y oscurecer su nombre, reservando ese derecho al hombre como exclusivo poseedor del vasto campo de la inteligencia" (Casanova, 1871: 24).

En el cierre de su texto, Eduvigis Casanova dedica unas palabras a exponer por qué el estilo de enseñanza sugerido por ella no se opone a la formación religiosa de las mujeres. En ese sentido, menciona que todos deberían desarrollar los talentos brindados por Dios, y que una amplia instrucción evitaría malinterpretar la religión, distanciando a las personas de supersticiones y creencias mal fundadas. Esta última aclaración de su artículo responde, por una parte, al hecho de que las intelectuales de este movimiento no buscaron oponer ciencia y religión -como ya explicábamos- $y$, por otra, a que cuando escribe la pedagoga se está en medio de otro debate: la laicización del Estado. Quizás por estrategia, o tal vez por convicción, Casanova decide no profundizar en esta otra controversia y mantiene una mirada conciliadora entre ambas perspectivas. Creemos que la escritora tiene claro que su irrupción en el espacio público a través de este texto no puede pronunciarse sobre todo tipo de temáticas, limitándose a atenerse solo a ciertos puntos sobre los cuales goza de cierta autoridad. Es en esta y otras partes de su texto donde se observa que la autora pareciera estar trazando espacios propios de acuerdo a lo planteado por Nancy Fraser (1992), ya que se distancia de un debate principal (la 
laicización del Estado) instalado en el espacio público, que desde la perspectiva imperante, hegemónica, sería imposible de evadir, sobre todo si se habla de educación.

La segunda publicación que debería considerarse un hito en este movimiento a favor de una instrucción científica para las mujeres, es la mencionada traducción de "Subjection of Women" de John Stuart Mill realizada por Martina Barros Borgoño en 1872.

En su prólogo la instruida sobrina de Diego Barros Arana realiza una crítica a la falta de derechos sociales para las mujeres, y para justificar su exigencia, recuerda diversos casos en que estas han superado a los hombres en talento y contribución al saber: "Recuerdo de todas aquellas controversias que aparecieron con el Renacimiento y en que no fue raro ver tomar parte a mujeres cuyo talento y elocuencia ha dejado un nombre en las cátedras de la filosofía y las ciencias sociales" (Barros, 1872: 115).

Luego acusa al tipo de educación impartida como la causa de la distinción arbitraría que se hace entre los dos sexos:

"A la sombra de la fuerza se ha sostenido merced a una educación viciosa que hace al hombre mirarse desde la cuna como un ser superior a la mujer (...). Se la somete a un sistema de educación cuya primera base viene a descansar sobre la misma idea de una inferioridad que de ese modo echa raíces en su espíritu" (Barros, 1872: 116).

Martina Barros explica que la naturaleza de la mujer, así como la del hombre, es la de ser libre y, en consecuencia, cada uno debe elegir qué hacer con su vida, lo que se vincula directamente con la discusión sobre la profesionalización de las mujeres. "Que la mujer sea libre para seguir el camino por donde la guían los instintos de su corazón y las aspiraciones de su espíritu" (Barros, 1872: 120) o "darle a la mujer la misma libertad que tiene el hombre para emplear sus facultades en el sentido que mejor le cuadre, es decir, darle la libertad de instrucción y la libertad para hacer uso de sus conocimientos" (Barros, 1872: 121), son algunos de los potentes llamados de esta autora con tan solo veintidós años de edad.

Lamentablemente, la sociedad no estaba preparada aún para recibir afirmaciones sin filtro de parte de una mujer, puesto que la censura social que recibió Martina Barros fue tan recia, que la llevó a guardar silencio durante los próximos 45 años, como ella misma expresa en sus memorias (Barros, 1942) ${ }^{9}$. Este es un ejemplo de los efectos que podía acarrear el no empleo de las "tretas del débil", como las plantea Josefina Ludmer (1985), y de utilizar, en cambio, una escritura no acorde a la posición subordinada y marginal de las mujeres (Ludmer, 1985).

Un tercer hito relevante que dio fuerzas al proceso analizado, se vincula a que las mujeres comenzaron a comunicar materias científicas en la prensa chilena. En este sentido, Revista de Valparaíso, primera en ser dirigida por una mujer (Rosario Orrego), fue trascendental, puesto que se constituyó como un espacio para que ellas pudieran

'La siguiente publicación de Martina Barros data de 1917, y consistió en un ensayo titulado "El voto femenino". 
pronunciarse sobre ciencia. Una de las primeras en firmar con su nombre y difundir conocimientos científicos derechamente en nuestro país fue Regina Uribe, hija de Rosario Orrego, quien en 1873 publicó en las páginas de este proyecto el texto "Idea del cosmos". Este acontecimiento es fundamental, puesto que si aplicamos las ideas de James Secord (2004) presentadas en el primer apartado de este artículo, podemos afirmar que en ese entonces algunas chilenas ya se encontraban contribuyendo claramente con la producción de conocimiento científico. Este autor, recordemos, postula que es imposible separar el hacer ciencia con comunicar esta, porque la transmisión del conocimiento es parte del proceso mismo de producción del saber. Los nuevos enfoques desarrollados por la historia de la ciencia, como expusimos al inicio de este trabajo, permiten incluir a los sujetos periféricos o "inexpertos" como agentes activos de generación del conocimiento (Nieto-Galán, 2011).

Esto último nos lleva a inferir que al traducir o escribir sobre asuntos científicos en la década de 1870, las mujeres estaban produciendo ciencia y, en consecuencia, estaban preparadas para comprender y estudiar disciplinas científicas.

Un siguiente hito del proceso que facultó a las chilenas a obtener el derecho a educarse científica y profesionalmente, se compone de las solicitudes de directoras de colegios femeninos (privados) a la comisión universitaria, para que las alumnas pudieran validar sus exámenes de bachillerato e ingresar a la educación superior. Antonia Tarragó fue una de las protagonistas de este acometido. Como ya hemos adelantado, siendo directora del colegio privado Santa Teresa, en 1872 elevó esta solicitud. Su petición no consiguió una respuesta favorable por parte de las autoridades, pero activó el debate en cuestión en las páginas de la prensa. La segunda protagonista de este hito fue Isabel Le Brun, quien siendo la directora del Colegio femenino La Recoleta, en 1876 elevó nuevamente la solicitud en 1876, que finalmente sí sería escuchada por las autoridades. Su estrategia retórica es interesante, ya que en vez de imponerse en su texto con tono pertinaz, explica que la razón por la cual se dirige a las autoridades es porque desconoce "de alguna disposición universitaria que reglamente los exámenes de las señoritas que aspiren a garantizar con certificados legales sus aptitudes para optar a grados superiores" (Le Brun, 1877: s/p). La pedagoga pide se le notifique de la existencia de dicha disposición, sabiendo, por supuesto, que no la hay. Luego propone que en el caso de que hubiese un "vacío" legal al respecto, se apliquen las mismas normativas que rigen a los colegios particulares, sin especificar que se refiere a los masculinos, y dando a entender que el suyo -uno femenino- no era distinto a estos últimos. Por consiguiente, de manera muy astuta, Le Brun se sirve de la apertura que las mismas leyes le proporcionan para lograr su objetivo. Por una parte, afirma que ella hablaba en nombre de los padres de estas niñas, quienes la habrían exhortado constantemente "para que dé la garantía que me propongo en mi solicitud de exámenes ante las comisiones universitarias" (Le Brun, 1877: s/p), despojándose, con ello, de la responsabilidad de su alegato. Por otra, explica a las autoridades que su trabajo sería más sencillo si éstas se manifestasen "sobre algún nuevo plan de estudios dictado para la instrucción sólida de la mujer, el que sería seguido en mi colegio llenando puntualmente todas las exigencias" (Le Brun, 1877: s/p), aludiendo a que no existían planes específicos para las alumnas de secundaria estipulados por el 
Estado y manifestando la negligencia de los encargados a través de un tono delicado que no hiere. A continuación, la pedagoga aclara que hasta la fecha -al no existir dicho programa- ella ha dirigido su establecimiento de acuerdo al plan del Instituto Nacional o Liceo emblemático masculino, revelando que había tomado las precauciones necesarias para defender a sus alumnas en el caso de que fueran sometidas a la evaluación. El texto de Le Brun revela que ella tenía pleno conocimiento de los contraargumentos que podría recibir, por lo que parece preparada para vencer cada uno de ellos, parapetándose especialmente con la idea de que si no existía una normativa que reglamentara o rechazara la validación de exámenes de mujeres que quisieran acceder a estudios superiores, entonces no había impedimentos para que ellas pudieran entrar a la universidad. Vemos también cómo ella rompe los límites, instruyendo a sus alumnas con el mismo plan de estudio que el Instituto Nacional, ya que si se presentaba la posibilidad de que sus alumnas fueran examinadas por la comisión, ellas debían demostrar tener plena capacidad para acceder a los estudios universitarios.

En definitiva, el texto de Le Brun también evidencia el uso de recursos retóricos en consonancia con las "tretas del débil" de Ludmer (1985), ya que si bien es posible dilucidar una vasta preparación en materia política y legislativa, cuida su discurso con frases menos duras e intenta quitarse de encima la entera responsabilidad de su solicitud.

Las habilidades argumentativas de Le Brun, la cobertura de la prensa y la opinión pública brindaron el impulso final para que el Ministro de Instrucción se viera obligado a autorizar lo que las pedagogas exigían. Tal como señala Sánchez (2006), "este nuevo fracaso en los intentos por ensanchar la instrucción de la mujer no pasó desapercibido para la prensa. El Independiente fue el primero que cubrió la noticia, al dar cuenta en su edición del 16 de diciembre de 1876 que tenía conocimiento de la solicitud de Le Brun" (Sánchez, 2006:8). Un mes después, levantaría la voz de alarma La República, dedicando en su edición del 16 de enero de 1877 un amplio espacio para cubrir la solicitud de la pedagoga, así como el informe sobre el estado de su colegio y las sesiones del Consejo dedicadas al tema (Sánchez, 2006). El revuelo que ocasionó este periódico activó a los medios restantes, forzando finalmente a Amunátegui a firmar el famoso Decreto el 6 de febrero de ese año.

Un nuevo hito en el proceso analizado es la fundación del periódico La Mujer de Lucrecia Undurraga. Este proyecto, como ya decíamos, fue enteramente dirigido y producido por mujeres y puede considerarse el primero con estas características en Chile (Ramírez y Ulloa, 2018). Circuló entre mayo y noviembre de 1877 y es un respaldo primordial para enterarnos de que la acción de este grupo de intelectuales no concluyó tras la Ley Amunátegui. En sus páginas nos enteramos de las nuevas preocupaciones que afloraron tras esta primera victoria. La creación de liceos públicos para las mujeres, la necesidad de implementar nuevos planes de estudio para los establecimientos femeninos y, sobre todo, el tipo de instrucción que convenía a las niñas, serán los temas transversales de este periódico. El debate en esta publicación otorgó relevancia al rol de las mujeres en el ámbito científico, puesto que una vez aprobado el acceso de ellas a la universidad, la mayoría de las diferencias radicaban ahora en determinar qué asignaturas científicas eran las apropiadas para ellas. A partir de esta publicación, además, puede 
constatarse una toma de conciencia colectiva de que ahora eran las mismas mujeres las que debían reclamar por sus derechos, como puede leerse en su prospecto:

"Queremos que sea ella misma -la mujer- quien defienda sus desatendidos intereses; que sea su voz elocuente y persuasiva, como es siempre la voz del que sufre, la que reclame sus derechos desconocidos y ponga en evidencia su falsa situación como miembro de una sociedad que pretende cubrirse con el brillante ropaje de la civilización y la cultura" (Undurraga, 1877: s/p).

La Mujer fue un espacio periodístico en el que ellas pudieron incluso auto-evaluar cómo habían llevado a cabo durante esos años el debate por la exigencia de derechos educativos. En sus páginas, por ejemplo, leemos un texto de Antonia Tarragó donde lo que la preocupa es la manera errónea en que se ha conducido el movimiento intelectual sobre la educación científica de la mujer. Para Tarragó el problema que ha obstaculizado el avance en esta materia radica en la falta de prudencia y verdad con la que se ha sostenido la discusión. Por lo que en su introducción dice que: "En el terreno de la ilustración de la mujer, unos se han propuesto ensalzar su capacidad hasta un grado insostenible, y otros la han deprimido tanto, que han querido hacerla descender del pedestal de inteligencia donde la colocó el Autor de la naturaleza” (Tarragó, 1877: s/p). Luego añade que: "las mejores causas pueden perderse no solo por llevar su defensa más allá de los límites de lo razonable y lo justo, sino también por restringir los títulos y derechos de su verdadera y legítima acción" (Tarragó, 1877: s/p). Su texto revela que ya para 1877, algunas intelectuales que lideraban el debate eran capaces de analizar y autoevaluar cómo se estaba llevando a cabo el movimiento y cuáles eran las falencias en la manera de sostener sus peticiones, lo que es a fin de cuentas una aprehensión propia del ejercicio de la política. Tarragó concluye que para lograr avances concretos en la materia se debe mantener una estrategia comunicacional que batalle con prudencia, porque cometer excesos no conduciría a nada:

"Así que la solución del problema de elevar a la mujer a las alturas de la ciencia, si es verdad que no puede aprovechar a todas, no lo es menos que la negación completa de tan legítima aspiración, a más de una grave injuria, envuelve el atropellamiento de un derecho y evidente y la traba de un gran progreso social" (Tarragó, 1877: s/p).

El último hito que consideramos importante para esta causa durante el siglo XIX, se vincula a una etapa en que las intelectuales ya han logrado graduarse de instituciones universitarias y se vuelven profesionales en materias científicas. La estrategia para sus peticiones, en ese sentido, muta, ya que las mujeres comenzaron a defender su derecho a desarrollarse científicamente desde su propia condición de expertas científicas. Un artículo que ejemplifica lo anterior es el que publica Margarita Práxedes Muñoz en Biblioteca Republicana en 1894. La autora era Bachiller en Ciencias Naturales por la 
Universidad de San Marcos (Perú), y en 1885 inició sus estudios de medicina en la Universidad de Chile. Su texto se titula "Diferencias en el volumen craneoscópico de los dos sexos", y lo dedica a Lucrecia Undurraga, dato que reafirma la configuración de una red entre las intelectuales que lideraban el movimiento.

En su colaboración en la prensa, Muñoz utilizó datos y metodología científica para apoyar la idea de que las mujeres son tan inteligentes como los hombres e igualmente dispuestas al estudio y ejercicio de las ciencias. Para ello citó estudios especializados en craneoscopia, tales como los de Broca y Vogt, al mismo tiempo aplicó conceptos técnicos e información que denota su preparación científica en el asunto, como puede leerse en este fragmento:

"Vista la deficiencia de datos que suministraba el ángulo facial de Camper, para apreciar debidamente el mayor o menor desarrollo de la inteligencia, consagra el primero las poderosas facultades de su espíritu y la indagación de este problema, el más importante de la moderna antropología y en el luminosísimo estudio que forma una de las más ricas joyas de sus trabajos sobre este ramo del saber, expone brillantemente los precisos resultados prácticos que se obtienen sustituyendo el ángulo facial de Camper por el esfenoidal o ángulo de la silla turca” (Muñoz, 1984:32).

Sus argumentos cobran mayor peso por el hecho de ser una experta en la materia y por ordenar sus ideas bajo una estructura desde la cual se suele discutir científicamente. De modo que para defender lo mismo que sus pares intentaron justificar durante las décadas anteriores, Muñoz apela al dato, así como a la metodología y lenguaje científicos:

"Si la mujer en las naciones cultas queda muy rezagada en el desarrollo de sus facultades intelectuales, fácilmente nos lo explica la ley biológica que preside el desenvolvimiento de los órganos, según la cual la actividad es condición indispensable del incremento de estos, de tal manera que cualquiera parte del organismo individual privada de ejercicio se entorpece, se atrofia y concluye a veces por quedar incapacitada completamente para llenar su función” (Muñoz, 1894: 34).

Con este último texto se puede inferir que si bien las mujeres generaron espacios propios y subalternos, como plantea Nancy Fraser (2004), dentro de estos ellas fueron trazando un camino hacia la participación en la esfera pública. Es interesante constatar, además, que para poder acceder a dicho espacio tuvieron que desprenderse paulatinamente de las estrategias discursivas que les sirvieron durante sus primeros pasos y adquirir herramientas (el lenguaje y la metodología científica hegemónicamente establecidas) que les permitiera desenvolverse con éxito en la dimensión pública.

\section{Conclusión.}


En los apartados anteriores hemos presentado los principales momentos y las características del proceso que protagonizó un conjunto de mujeres para conseguir el derecho a educarse científicamente en Chile. Específicamente, nos hemos centrado en la batalla que ellas lidiaron a través de la prensa durante el siglo XIX para lograr concientizar a las autoridades y a la sociedad de que las mujeres debían acceder a los estudios superiores y ejercer profesiones científicas. No hay duda de que la preparación en materia educativa, la adquisición de experiencia en asuntos políticos y sus conversaciones en salones y otro tipo de instancias, hubo de influir en la consecución de su propósito, pero la prensa fue uno de los medios más relevantes al momento de conseguir resultados concretos.

Se ha demostrado que ellas utilizaron diversas estrategias para pronunciarse sobre lo que les estaba vedado y para entrar en espacios que les eran negados. Entre todos esos recursos, el uso de la palabra se destaca, ya que fue la primera arma con la que contaron para avanzar en la materia. Por ello la irrupción de la mujer en la prensa, en el campo literario y en el mundo científico se produjo en forma simultánea y cooperativa. Las intelectuales que dieron la pelea obtuvieron resultados ocupando estos tres espacios sincrónicamente. El acceso a los estudios y profesiones científicas no habría sido posible sin el manejo de la pluma y sin la publicación de sus ideas en las páginas periodísticas, por lo que saber expresarse y utilizar a su favor el lenguaje fueron tácticas fundamentales para abrirse camino hacia el ámbito científico.

Un aspecto que se esboza en este artículo, pero que consideramos relevante profundizar en un futuro estudio histórico de este proceso, es el examen y valoración de la vocación y talento literario de estas mujeres, que las facultó a manejar el lenguaje de manera exitosa y favorable hacia su causa, análisis que requeriría el empleo de perspectivas teorías interdisciplinarias, incluyendo a la literatura. La mayoría de estas mujeres escribió y publicó obras literarias en la prensa, lo que nos permite deducir que la literatura pudo ser una herramienta que les posibilitó asomarse en el espacio público y afianzar su rol dentro de este. La expresión literaria en sí pudo funcionar como recurso para poder exponer públicamente sus opiniones, sin hacerse responsables de las consecuencias sociales que estas provocaban, puesto que podían justificarse a través de la ficción y del escenario virtual que genera este tipo de textos. Las estrategias que utilizaron las mujeres, como hemos demostrado anteriormente, se fundamentaron en tácticas y habilidades discursivas, pero adicional a ello, el uso de la imaginación y la capacidad de representar virtualmente la realidad también pudieron contribuir a la causa, por lo que el análisis de sus trabajos literarios y cómo estos se relacionan e inciden en sus textos de carácter científico, debe ser atendido con mayor detención.

Otro aspecto que tampoco agotamos en este trabajo y que consideramos necesario explorar para una mayor comprensión histórica del proceso descrito, consiste en la identificación en detalle de las redes que construyeron estas mujeres, tanto con otras intelectuales dentro y fuera de Chile, como con varones. Creemos que rastrear la formación autodidacta que ellas recibieron en espacios íntimos, así como los círculos y tertulias a las que asistieron, puede ser una vía de entrada para tejer esta red y para hallar nuevos hilos de desarrollo de este fenómeno. Es posible comprobar que algunas de 
las intelectuales citadas durante este artículo intercambiaron ideas con otras renombradas que lideraban procesos semejantes en el extranjero, como mencionamos en el desarrollo de este trabajo. Así mismo, tenemos datos que constatan que algunos varones fueron eslabones claves para que a ellas se les respetara el derecho a educarse. Máximo Ramón Lira y Eugenio María de Hostos defendieron públicamente en la prensa la solicitud de Tarragó10; Rodolfo Echeverría e Indalicio Segundo Díaz apoyaron el proyecto periodístico de Lucrecia Undurraga dedicado especialmente a transmitir las ideas del movimiento en pro de la educación y mejoramiento social de las mujeres en 1877; Ernesto Turenne publicó varios textos en favor de la causa, tal como "Profesiones científicas para la mujer" en Revista Chilena en 1877; los poetas Santiago Escuti Orrego, J. Aníbal Frías, Rafael Luis Solar y Francisco A. Subercaseaux, se consideraron colegas de varias escritoras y las invitaron a publicar en revistas y periódicos que ellos dirigían, y colaboraron en los proyectos que ellas lideraban ${ }^{11}$, A ellos se podrían sumar aquellos hombres que de manera generosa y desinteresada transmitieron sus conocimientos a algunas mujeres en espacios íntimos, como fue el caso de Diego Barros Arana y su sobrina Martina. Para lograr este propósito, la prensa es una fuente efectiva, que junto a otros documentos, tales como cartas, diarios de vida, etc., posibilitarían armar un corpus para rastrear estas redes. Este propósito, sin embargo, tendrá que lidiar con los obstáculos para hallar algunos de estos materiales que hasta nuestros días permanece fuera de los archivos.

Ambas perspectivas recién presentadas son piezas pendientesde nuestro análisis, que de efectuarse, complementarían un trabajo como este y ayudarían a generar una comprensión más acabada del proceso aquí estudiado.

\section{Bibliografía}

Acevedo, María Luisa (1889): "Sobre los estudios que convienen a la mujer", en Revista del Progreso, Tomo 3, p. 110-113.

Amunátegui, Miguel (1877): “Decreto Supremo de Chile N547”, en Anales de la Universidad de Chile, No. 52, p. 34.

Barros, Martina (1872): "La esclavitud de la mujer" (Prólogo), en Revista de Santiago, Tomo 2, p. 112-124.

\footnotetext{
10 Lira, además de político y diplomático, dedicó gran parte de su vida al periodismo. Tuvo una conversión desde el conservadurismo al liberalismo, que provocó polémica en la opinión pública. Fue en su rol de redactor principal de El Independiente que apoyó la causa de Antonia Tarragó. Eugenio María de Hostos fue un reconocido intelectual puertorriqueño, graduado de derecho y filosofía en Europa, y que vino a Chile a desempeñarse en el ámbito académico y a propagar sus ideas liberales. Trabajó como docente en la Universidad de Chile y fue el primer rector del Liceo Miguel Luis Amunátegui.

${ }^{11}$ Para profundizar sobre este tema, véase: Ramírez y Ulloa, 2018.
} 
Revista Punto Género № 12. Diciembre de 2019

ISSN 0719-0417 / 1 - 20

Barros, Martina (1942): Recuerdos de mi vida. Santiago: Orbe.

Bowler, Peter e Ivan Rhys Morus (2005): Making Modern Science. Chicago: The University of Chicago Press, pp. 487-509.

Casanova, Eduvigis (1871): Educación de la mujer. Santiago: Imprenta de la Patria.

Casanova, Eduvigis (1876): "Reflexiones sobre la educación pública de la mujer en Chile: Escuelas públicas de niñas", en La brisa de Chile, No.5, s/p.

De la Barra, Maipina (1885): “España”, en La Patria, s/p.

Fraser, Nancy (1992): Repensando la esfera pública. Una contribución a la crítica de la democracia actualmente existente, en Habermas and thePublic Sphere, ed. Craig Calhoun. London: The MIT Press, p. 4-9.

Kottow, Andrea (2013): "Feminismo y femineidad: Escritura y género en las primeras escritoras feministas en Chile", en Atenea, No. 508, p. 151-169.

Muñoz, Margarita Práxedes (1894): "Diferencias del volumen craneoscópico de los dos sexos", en Biblioteca Republicana, No.1, p. 32-34.

Lassabe, Celeste (1890): “Nuestra primera palabra”, en La Familia, No. 1, p. 1.

Latour, Bruno (1987): Science in Action. How to Follow Scientists and Engineers through Society.Harvard University Press.

Le Brun, Isabel (1877): "Solicitud e informe", en La República, p. 2.

Lipphardt, Veronika y David Ludwig (2011): "Knowledge Transfer and Science Transfer", en European History Online (EGO). Mainz: Institute of European History (IEG). URL: http://ieg-ego.eu/en/threads/theories-and-methods/knowledge-transfer/veronika-lipphardtdavid-ludwig-knowledge-transfer-and-science-transferr [02-03-2018]

Ludmer, Josefina (1985): “Las tretas del débil”, en La sartén por el mango. Puerto Rico: Ediciones El Huracán.URL: https://literaturaanimada.files.wordpress.com/2014/03/ludmertretas-del-dc3a9bil.pdf [20-11-2019]

Montero, Claudia (2018): Y también hicieron periódicos: Cien años de prensa de mujeres en Chile (1850-1950). Santiago: Hueders. 
Revista Punto Género № 12. Diciembre de 2019

ISSN 0719-0417 / 1 - 20

Muñoz, Margarita P. (1894): "Diferencias en el volumen craneoscópico de los dos sexos", en Biblioteca Republicana, No. 1, p. 32-34.

Nieto-Galan, Agustí (2011): Los públicos de la ciencia: Expertos y profanes a través de la historia. Madrid: Marcial Pons.

Opitz, Donald (2015): Domesticity in the Making of Modern Science. Palgrave Macmillan.

Ossandón, Carlos y Santa Cruz, Eduardo (2005): El estallido de las formas. Chile en los albores de la cultura de masas. Santiago: LOM.

Pinochet, Eurídice (1889): "Aniversario de una academia literaria”, en La República: Publicación literaria, No. 2, p. 13.

Ramírez, Verónica (2016): "Ciencia y mujer: aproximación a un estudio de las primeras divulgadoras de la ciencia en Chile", en Cuadernos de Historia Cultural, No. 5, p. 77-106.

Ramírez, Verónica, Manuel Romo y Carla Ulloa (2017): Antología crítica de mujeres en la prensa chilena del siglo XIX. Santiago: Cuarto Propio.

Ramírez, Verónica y Carla Ulloa (2018): La Mujer (1877): primer periódico de mujeres en Chile. Santiago: Editorial Cuarto Propio.

Renn, Jürgen (2015): "From the History of Science to the History of Knowledge - and Back", en Centaurus, No. 57, pp. 37-53.

Sánchez, Karin (2006): "El ingreso de la mujer a la universidad y los cambios en la costumbre por medio de la ley 1872-1877", en Historia, Vol. 2, No.39, p. 497-529.

Secord, James (2004): “Knowledge in Transit”, en Isis, No. 95, p. 654-672.

Stuven, Ana María (2010): Historia de las mujeres en Chile. Santiago: Taurus.

Tarragó, Antonia (1877): "Ilustración superior de la mujer", en La Mujer, No. 1, s/p.

Ulloa, Carla(2017): "Lucrecia Undurraga y el periodismo liberal de mujeres en el Chile Decimonónico: La Brisa de Chile (1875-1876) y La Mujer (1877)", en Contreras, Landeros y Ulloa: Escritoras chilenas del siglo XIX: su incorporación pionera a la esfera pública y el campo cultural. Santiago: RIL Editores.

Ulrica (1893): "Inconsecuencias del hombre apreciadas por una de sus hermanas", en La Verdad, No. 16, s/p. 
Revista Punto Género № 12. Diciembre de 2019

ISSN 0719-0417 / 1 - 20

Undurraga, Lucrecia (1877): "Prospecto-circular", en La Mujer, p. 1.

Uribe, Regina (1873): "Idea del cosmos”, en Revista de Valparaíso, Tomo 1, p. 250-251. 